Presented at Micro Bunches Workshop

Upton, NY, September 28-30, 1995

\title{
Design of the Muon Collider Isochronous Storage Ring Lattice
}

\author{
D. Trbojevic, K.Y. Ng, " E.D. Courant, S.Y. Lee, ${ }^{\dagger}$ \\ C. Johnstone," J. Gallardo, B. Palmer, and S. Tepikian \\ Brookhaven National Laboratory, Upton, Long Island, NY 11973.
}

\begin{abstract}
The muon collider would extend limitations of the $e^{+} e^{-}$colliders and provide new physics potentials with a possible discovery of the heavy Higgs bosons. At the maximum energy of $2 \mathrm{TeV}$ the projected luminosity is of the order of $10^{35} \mathrm{~cm}^{-2} \mathrm{~s}^{-1}$. The colliding $\mu^{+} \mu^{-}$bunches have to be focused to a very small transverse size of few tenths of $\mu \mathrm{m}$ which is accomplished by the betatron functions at the crossing point of $\beta^{*}=3 \mathrm{~mm}$. This requires the longitudinal space of the same length $3 \mathrm{~mm}$. These very short bunches at $2 \mathrm{TeV}$ could circulate only in a quasi-isochronous storage ring where the momentum compaction is very close to zero. We report on a design of the muon collider isochronous lattice. The momentum compaction is brought to zero by having the average value of the dispersion function through dipoles equal to zero. This has been accomplished by a combination of the FODO cells together with a low beta insertion. The dispersion function oscillates between negative and positive values.
\end{abstract}

Typeset Using REVTEX

*Fermi National Accelerator Laboratory, P.O.Box 500, Batavia, Il 60510

'Permanent address: Department of Physics, Indiana University, Bloomington, IN 47405.

IOperated by the Universities Research Association, Inc., under contract with the U. S. Department of Energy. 


\section{INTRODUCTION}

A muon collider will have two $\mu^{+} \mu^{-}$bunches in the same ring with $210^{12}$ particles per bunch. The luminosity could be presented as:

$$
\mathcal{L}=\frac{f_{\text {rep }} n_{b} N_{\mu}^{2}}{4 \pi \beta^{*} \epsilon_{N} \gamma}=10^{35} \mathrm{~cm}^{-2} \mathrm{~s}^{-1}
$$

where $n_{b}$ is the number of bunches, $f_{\text {rep }}$ is the repetition frequency, $N_{\mu}$ is the bunch population, $\gamma$ is the Lorentz relativistic factor, and $\varepsilon_{N}$ is the normalized tranverse emittance. Table I represents the basic parameters of the $2 \mathrm{TeV}$ muon collider:

TABLE L Basic Parameters of the $2 \mathrm{TeV}$ Muon collider

\begin{tabular}{||l|lr}
\hline \hline Energy & 2000 & $\mathrm{GeV}$ \\
\hline Normalized Emittance $\varepsilon_{N}$ & 50 & $\mathrm{~mm}$ mrad \\
\hline Beta Functions at the IP $\beta^{*}$ & 0.003 & $\mathrm{~m}$ \\
\hline Length of the bunch $\sigma_{z}$ rms & 0.003 & $\mathrm{~m}$ \\
\hline Momentum Spread $\frac{\Delta E}{\varepsilon}$ & 0.5 & $\%$ \\
\hline Repetition Rate $f$ & 30 & $\mathrm{~Hz}$ \\
\hline Effective number of Turns $n_{s}$ & 900 & \\
\hline Number of Bunches & 1 & \\
\hline Number of Muons per Bunch $n_{b}$ & $2 * 10^{12}$ \\
\hline \hline
\end{tabular}

The minimum values of the betatron functions at the beam collision point require very strong and large aperture quadrupoles with the maximum values of the betatron functions of the order of tenths to hundreds kilometers. The chromaticity in the interaction regions has unusual large values. The chromaticity has to be compensated by separate sextupoles. To cancel the sextupole induced second order amplitude dependent ture spread as well as the higher order aberrations due to the interaction between the sextupoles and the interaction quadrupoles the sextupoles have to be be located around the interaction region with $n \pi$ phase difference with respect to the the interaction quadrupoles. There should be a $\pi$ betatron phase difference [1, 2] in each $\Phi_{x}$ and $\Phi_{y}$ betatron phases between each pair of sextupoles. The best solution [1] is with two pairs of horizontal and vertical sextupoles on each side of the interaction region. This report will present mostly the isochronous part of the collider ring lattice. 


\section{A. Equations for the Longitudinal Motion}

A paricle motion in the longitudinal phase space depends on the particles time of arrival at the RF cavities and to the first order could be presented as:

$$
\begin{gathered}
\frac{\Delta T}{T_{0}}=\frac{\Delta C}{C_{0}}-\frac{\Delta v}{v} . \\
\frac{\Delta T}{T_{0}}=\eta \frac{\Delta p}{p_{0}}=\left(\alpha_{0}-\frac{1}{\gamma^{2}}\right) \frac{\Delta p}{p_{0}} .
\end{gathered}
$$

where $T_{0}$ is the time of the arrival of the reference particle, $\Delta T$ and $\Delta p$ are the time and momentum deviation, respectively, of the off-momentum particle relative to the synchronous particle with the momentum $p_{0}$, where $\eta$ is the "phase slip" slip factor, $\gamma$ is the Lorentz relativistic factor, while $\alpha_{0}$ is to the first order momentum compaction of the lattice. The transition $\gamma_{t}$ is defined as $\alpha_{0}=\frac{1}{\gamma_{z}^{2}}$ The revolution time of particles with different momenta in the regular FODO lattice depend on their energy. In the isochronous storage ring the revolution time of particles with different momenta does not depend to their energy. When a value of the momentum compaction is close to zero then the second order quantities have to be considered. The beam life time depends on stability of the longitudinal phase space. It has been shown, $[5,3,6]$ that conditions for the stable longitudinal phase space could be set. Also, the influence of the nonlinear terms in $\delta=\frac{\Delta p}{p_{0}}$ on the stability of the longitudinal phase space could be controlled. Sextupoles and other higher order elements could be used to help the control of the longitudinal phase space. The longitudinal phase space dependence on the nonlinear terms in $\delta$ is due to variations of the dispersion function and of the momentum compaction $\alpha$ on $\delta$. A difference in orbit length $\Delta C$ of the particle with a momentum offset depends on the higher order of the momentum [5] $\delta$ as:

$$
\frac{\Delta C}{C_{0}}=\alpha_{0} \delta\left[1+\alpha_{1} \delta+\varepsilon\left(\delta^{2}\right)\right]
$$

The phase slip factor $\eta$ could be presented as [5]:

$$
\eta=\eta_{0}+\eta_{1} \delta+\varepsilon\left(\delta^{2}\right)
$$

where:

$$
\eta_{0}=\alpha_{0}-\frac{1}{\gamma^{2}}=\frac{1}{\gamma_{t}^{2}}-\frac{1}{\gamma^{2}}
$$


and

$$
\eta_{1}=\alpha_{0} \alpha_{1}+\frac{3}{2} \frac{3^{2}}{\eta^{2}}-\frac{\eta_{0}}{?^{2}}
$$

The dispersion function is defined as:

$$
D_{=}=\frac{\Delta x}{\delta}=D_{0}+D_{1} \delta+\varepsilon\left(\varepsilon^{2}\right)
$$

The momentum compaction defined to the lowest order in $\dot{\varepsilon}$ is related to the horizontal dispersion function $D_{0}$ by

$$
\alpha_{0}=\frac{1}{C_{0}} \oint \frac{D_{0}(s)}{\rho(s)} d s,
$$

where $\rho$ is the radius of curvature and $s$ is the longitudinal path length measured along the reference orbit with a circumference $C_{0}$.

When the phase-slip factor is equal to zero then $\gamma$ is equal $\gamma t$. This condition would correspond to the transition.

Vladimirski and Tarasov [8] introduced reverse bends in an accelerator lattice and succeeded in getting negative orbit-length increase with momentum, thus pushing the transition energy to infinity. There was recently another proposal by the UCLA [3] group on the isochronous ring with the reverse bends in the lattice. Teng [9] reported earlier that the same can be accomplished with a regular dipoles placed at locations where the dispersion is negative. For the thin dipole the momentum compaction to the first order is:

$$
\alpha=\sum_{i} \bar{D}_{i} \theta_{i}
$$

where $\theta_{i}$ is the bending angle of the $i$ th dipole and $\bar{D}_{i}$ is the average dispersion function at the dipole location. The condition for an isochronous storage ring lattice is to have average horizontal dispersion through most of the dipoles equal to zero: $\left.\sum_{i} \bar{D}_{i} \theta_{i}\right|_{\text {dipole }}=0$; There are many variations of the Teng's method, which are usually termed the harmonic approach and high-tune approach $[10,12,13]$. This method will create a systematic stopband to induce dispersion-wave oscillations resulting in lower dynamical aperture. As we reported earlier [18] the quasi-isochronous storage ring can be designed without use of the reverse bends. Instead we use a combination of the FODO cell with a low beta insertion, or the $\pi$ module, with a dipole in the middle. This allows better compaction of the ring and reduces the maxima of the dispersion function. We had studied extensively the tranverse beam dynamics $[11,14,15,19]$ of this kind of lattice and showed that the latice is very stable and has very good tunability. Details of the design of the hasic modules could be found elsewhere [14]. 


\section{NORMALIZED DISPERSION SPACE}

The equation of motion to the second order in $\delta$ was presented [5] as:

$$
x^{\prime \prime}+\left(1+\frac{x}{\rho}\right)\left(\frac{1}{1+\delta}\right)\left(\frac{1}{\rho^{2}}-K_{1}\right) x=\left(1+\frac{x}{\rho}\right)\left(\frac{\delta}{1+\delta}\right)\left(\frac{1}{\rho}\right)+\frac{x^{\prime 2}+y^{\prime 2}}{2 \rho}-K_{2} \frac{1}{2}\left(x^{2}-y^{2}\right),
$$

after substitution [5]:

$$
x=D_{0} \delta+D_{1} \delta^{2}
$$

and $y=0$ the first order equation is presented as:

$$
D_{0}^{\prime \prime}+K_{x}(s) D_{0}=\frac{1}{\rho(s)}
$$

while the second order equation is:

$$
D_{1}^{\prime \prime}+K_{x} D_{1}=\frac{D_{0}^{\prime 2}}{2 \rho}-K_{1} D_{0}-\frac{1}{\rho}\left(1-\frac{D_{0}}{\rho}\right)^{2}-\frac{1}{2} K_{2} D_{0}^{2}
$$

where the prime denotes the derivative with respect to the longitudinal coordinate $s, \rho(s)$ is the local radius of curvature, and: $K_{1}=\frac{1}{B_{\rho}} \frac{\partial B_{y}}{\partial x}, K_{2}=\frac{1}{B_{\rho}} \frac{\partial^{2} B_{y}}{\partial^{2} x}$, and $K_{x}=$ $\frac{1}{\rho^{2}}-K_{1}$. The $K_{x}$ is the sum of the quadrupole $K_{1}$ and centrifugal focusing, while $K_{2}$ is the sextupole strength. The normalized dispersion function with components $\xi$ and $\chi$ is defined as,

$$
\xi=\sqrt{\beta_{x}} D^{\prime}-\frac{\beta_{x}^{\prime}}{2 \sqrt{\beta_{x}}} D=\mathcal{D} \cos \phi, \quad \chi=\frac{1}{\sqrt{\beta_{x}}} D=\mathcal{D} \sin \phi,
$$

where $\beta_{x}$ and $\beta_{x}^{\prime}$ are respectively the horizontal betatron amplitude function and its derivative [16], $\mathcal{D}$ is the norm or length of the normalized dispersion vector, and $\phi$ is identical to the horizontal Floquet betatron phase advance in the region where there is no dipole. In the thin-element approximation, in normalized $\xi-\chi$ space, the normalized dispersion vector changes by $\Delta \xi=\sqrt{\beta_{x}} \theta$ and $\Delta \chi=0$. Outside the dipole $(\rho=\infty)$, the dispersion function satisfies the homogeneous equation, so that $\mathcal{D}$ is an invariant, with $\xi$ and $\chi$ satisfying $\xi^{2}+\chi^{2}=\mathcal{D}^{2}$, which is a circle. The normalized dispersion vector advances by an angle $\phi$. This type of normalized dispersion plots has been successfully used in lattice design and beam-transfer line design. It has also been used to lower the emittance in the electron storage rings [4] and to design a low emittance isochronous electron ring [18]. 
The basic module of the the isochronous muon collider storage ring(IMCSR) is made of the FODO cells and $a \pi$ insertion. In the two FODO cells the dispersion function oscillates between negative and positive values within the dipoles providing zero value of the momentum compaction of the whole lattice. We use a reflective symmetry of all Courant-Snyder functions within the module with respect to the vertical $\chi$ axis in the normalized dispersion space. The reflection symmetry simplifies the analysis and optical matching considerably. Other details of this module have already been presented [11]. To build a module with a momentum compaction factor close to zero the dipoles should be placed in both positive and negative values of the $\chi$ axis of the $(\xi, \chi)$ normalized dispersion space. The dispersion function at the beginning of the FODO cell is prescribed with a negative value $D_{A}$ with $D_{A}^{\prime}=0$. As we emphasized earlier [11] the choice of $D_{A}$ is important to dispersion excursion and to the value of the momentum compaction $\alpha_{0}$.

A first example of the zero momentum compaction basic module of the ISMCSR is presented in figure 1 .

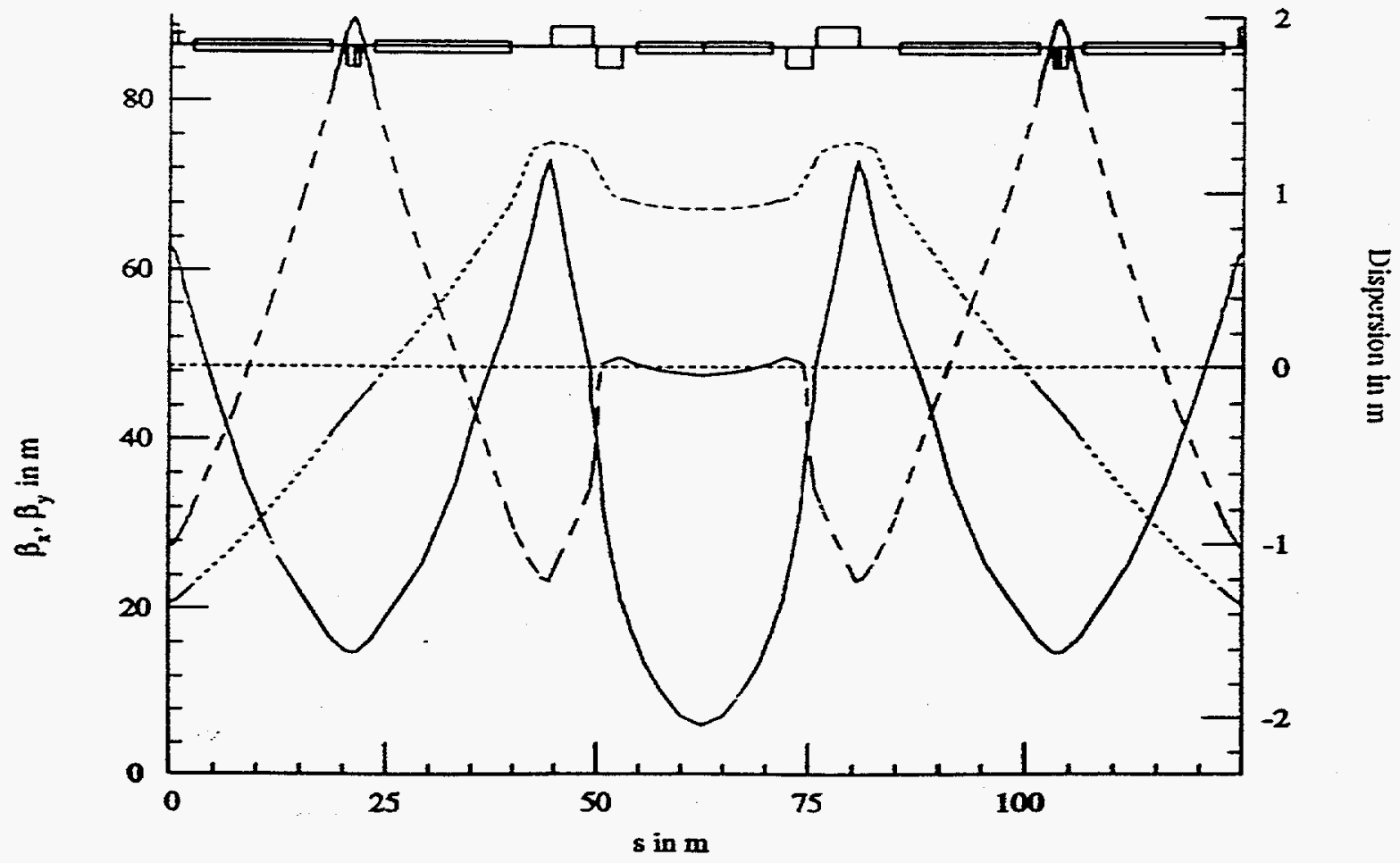

Dispersion max/min: $128722 / 1.33740 \mathrm{~m}$,

$\beta_{x}$ max/min $7326 / 596826 m, v_{x}=0.87896, \xi_{x}:-0.45$,

B, max/mix $89.962329385 m, v_{y}: 0.45932, \xi_{y}=0.23$,

$K=(000,=* * * * *)$

Modele langth: $1248779 m$

Toeal beod angle: $0.11219974 \mathrm{rad}$

Betatron Functions in the $\alpha=0$ basic module of the Muon Collider Storage Ring. 
The momentum compaction factor is set to zero for this particular block. It is important to note that the module could be tuned to whatever value of the momentum compaction $\alpha$ is needed to adjust the momentum compaction of the whole ring. This is to compensate for the momentum compaction of the interaction regions, extraction/injection regions, and zero dispersion blocks in the ring. The dipoles are $16 \mathrm{~m}$ long with the maximum magnetic field close to $9 \mathrm{~T}$. There is one $16 \mathrm{~m}$ long dipole per half cell as well as one dipole in the middle of the low beta insertion cell. Two FODO cells are bracketed by two quadrupoles- doublets which provide the low beta $\beta_{x}$ insertion part. The maxima of the betatron functions are $\beta_{x m a x}=73.26 \mathrm{~m}$ and $\beta_{y \max }=89.96 \mathrm{~m}$ with the dispersion function oscilating between $D_{x \max }=1.287 \mathrm{~m}$ and $D_{x \min }=-1.337 \mathrm{~m}$. The length of this module is $\sim 125 \mathrm{~m}$. The second order tune spread of the chromaticity sextupoles is very small:

$$
\nu_{x}=0.87896-1.33 \varepsilon_{x}+9.76 \varepsilon_{y} \quad \nu_{y}=0.45932+9.76 \varepsilon_{x}+24.4 \varepsilon_{y},
$$

where the $\varepsilon_{x, y}$ are the unnormalized emittances. The quadrupole lengths are $L_{1}=$ $1.6 \mathrm{~m}$ and $L_{2}=4.94 \mathrm{~m}$, and $L_{3}=3.05 \mathrm{~m}$, where the $L_{1}$ is a length of the FODO cell quadrupoles, while the $L_{2}$ and $L_{3}$ are the lengths of the $\pi$ insertion quadrupoles. The gradients in all quadrupoles are GF $=263.84 \mathrm{~T} / \mathrm{m}$ and $\mathrm{GD}=-205.36 \mathrm{~T} / \mathrm{m}$. The

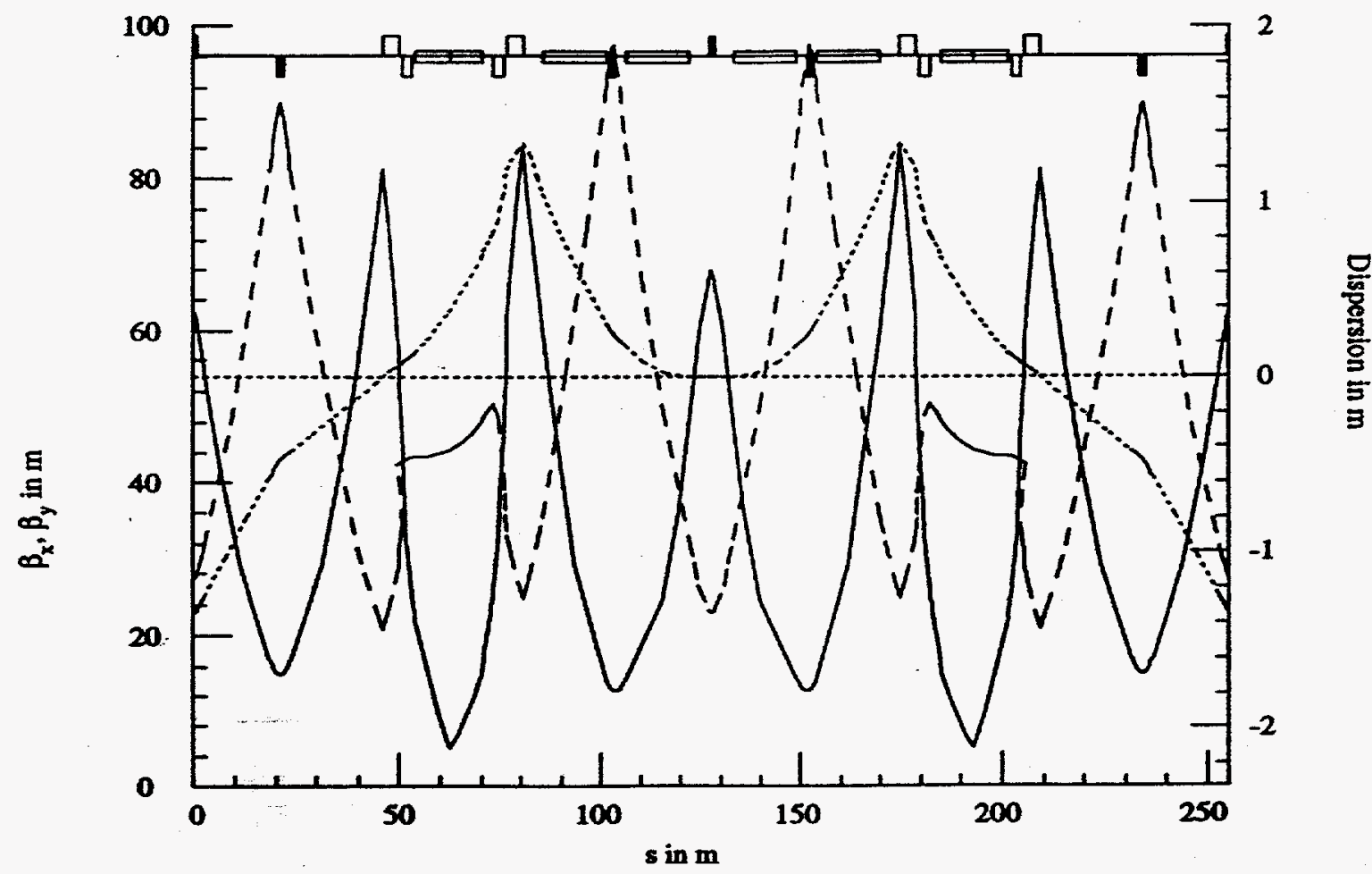

Dispersion max/min: $1.33509 / 1.33740 \mathrm{~m}$,

$B_{x}$ maxtmin: $8452 / 4.94518 \mathrm{~m}, v_{x}=1.87182, \xi_{x}:-1.43$,

$\beta_{y}$ maxtmin: $97.7520 .93837 \mathrm{~m}, v_{y}: 0.94906, \xi_{y}:-0.86$,

$\gamma_{i}:(72.03,0.00)$

Moduke kength: 255.1788m

Towal bend angle: $0.13463968 \mathrm{rad}$ 
second example of the basic module with the zero momentum compaction has two $9.45 \mathrm{~m}$ long dipoles per half FODO cell and one $9.45 \mathrm{~m}$ dipole within the $\pi$ part of the module.

The next module which provides the zero dispersion at the end of it, is presented in figure 2. This module is matched to the basic module with all betatron functions and could be used for the two injection-extraction or RF straight sections.

The interaction region is matched to the end of the 15 basic modules. We present in Figure 6 one of the initial solutions of the interaction region which does not fulfill the request for cancelation of the higher order aberrations but it does have reduced the second order tune spread due to chromaticity sextupoles.

FIG. 3.

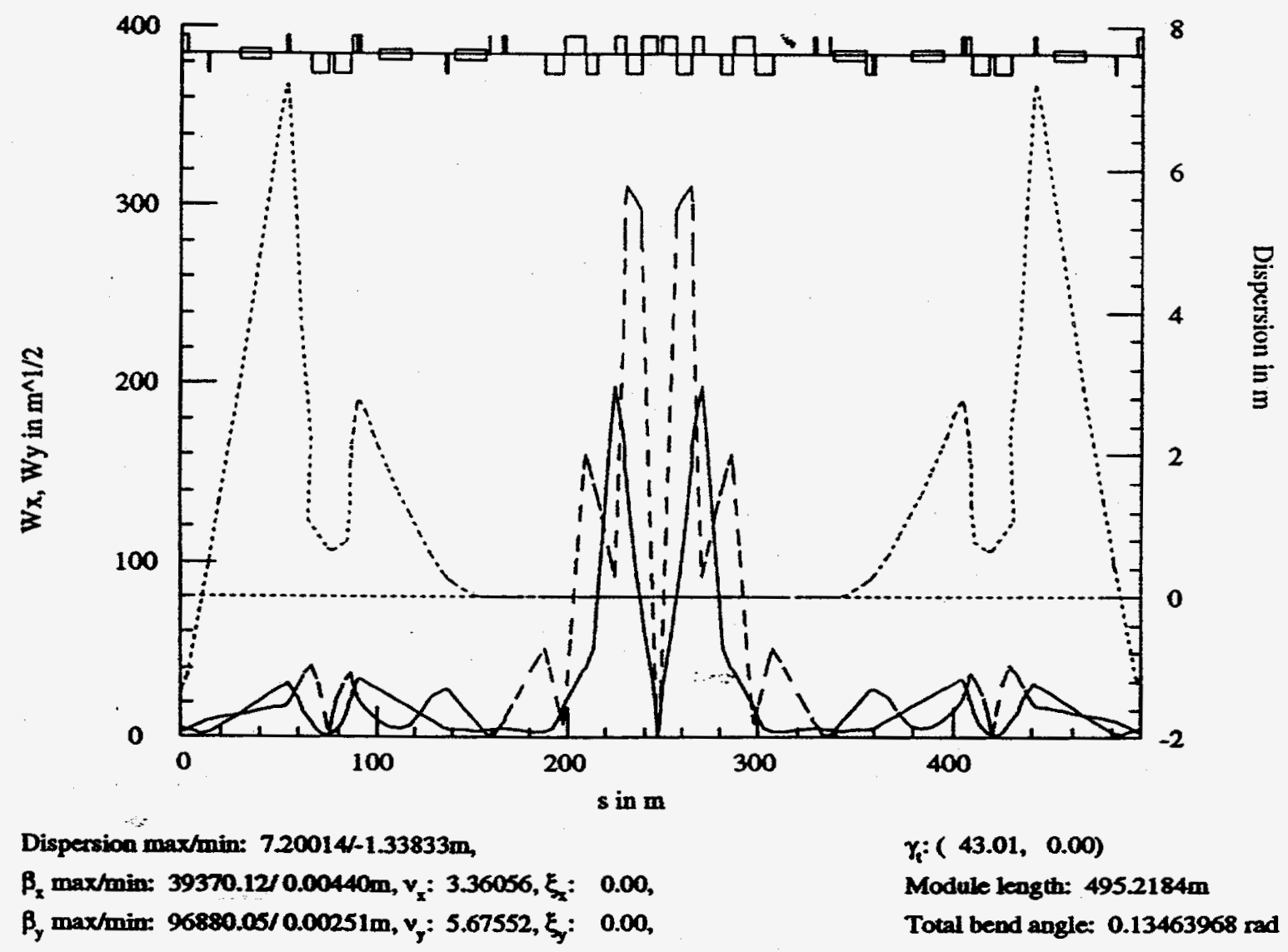

Betatron Functions in one of the First Solutions of the Interaction Region. 


\section{CONCLUSION}

We have presented the Muon Collider Isochronous Storage Ring lattice. The mometum compaction has been brought to a zero value by dispersion function oscilation between negative and positiove values through the dipoles. We applied our basic design principle of the flexible-momentum-compaction lattice [11] to construct the muon collider isochronous storage ring. We presented modules with the momentum compaction equal zero with either $16 \mathrm{~m}$ long dipoles (SSC dipole) or with $9.45 \mathrm{~m}$ long dipoles (RHIC dipoles). The interaction region has the zero dispersion. The present design assumes two fold symmetry with two interaction regions and two zero dispersion regions for the injection/extraction and RF. The most chalenging part of the collider design is the interaction region wich requires very strong focusing quadrupoles with values of the betatron functions at the collition point as low as $3 \mathrm{~mm}$. The final solution of the interaction region will fulfill a cancelation of the higher order optical aberrations, reduction of the second order tune spread, reduction of the triplet induced betatron wave, etc. We have presented a realistic design of the muon storage ring collider which could be easily scaled down with energy if it is required. The high luminosity of the proposed $\mu^{+} \mu^{-}$collider could be achieved with very short bunches $3 \mathrm{~mm}$ and with the very small transverse beam sizes at the collision point $\beta^{*}=3 \mathrm{~mm}$ which this storage ring design provides.

\section{REFERENCES}

[1] N.J. Walker, J. Irwin, and M. Woodley, Analysis of Higher Order Optical Aberrations in the SLC Final Focus using Lie Algebra Techniques, presented at the 1993 IEEE Particle Accelerator Conference,'Washington, D.C., May 17-20, 1993.

[2] M. Donald, R. Helm, J. Irwin, H. Moshammer, E. Forest, D. Robin, A. Zholents, and M. Sullivan,Localized Chromaticity Correction of the Low-Beta Insertions in Storage Rings, presented at the 1993 IEEE Particle Accelerator Conference, Washington, D.C., May 17-20, 1993.

[3] D. Robin, E. Forest, C. Pellegrini, and A. Amiry, Quasi-isochronous storage rings, Physical Review E, Vol. 48, No.3, September 1993, pp. 2149-2156.

[4] D. Trbojevic and E. D. Courant, Low Emittance Lattices for Electron Storage Ring Revisited, Fourth European Accelerator Conference, London, June 27 to July 1, pp. 1000., 1994.

[5] J.P. Shan, S.G. Peggs, and S.A. Bogacz, Analytical Evaluation of the Second Order Momentum Compaction Factor and Comparison with MAD Results, Particle 
Accelerators, 1994, Vol.45, pp. 1-16.

[6] A. Amiry, C. Pellegrini, E. Forest, and D. Robin. Study of Magnetic Lattice for a Quasi-Isochronous Ring, Particle Accelerators. 1994. Vol 44. pp. 65-76.

[7] W.W. Lee and L.C. Teng, Proc. Sth Int. Conf. on High-energy Accelerators. CERN, pp. 327-330 (1971).

[8] V.V. Vladimirski and E.K. Tarasov, Theoretical Problems of the Ring Accelerators, USSR Academy of Sciences, Moscow (195j).

[9] L.C. Teng, Infinite Transition-energy Synchrotron Lattice using $\pi$-straight Section, Part. Acc. 4, pp. 81-85 (1972).

[10] R. Gupta and J.I.M. Botman, High Transition Energy Magnet Lattices, IEEE Trans. Nucl. Sci. NS-32, 2308 (1985).

[11] S.Y. Lee, K.Y. Ng, and D. Trbojevic, Minimizing dispersion in flexiblemomentum-compaction lattices, Physical Review E, Vol 48, No 4, October 1993,pp. 3040-3048.

[12] G. Guignard, A Lattice with no Transition and Largest Aperture, Proc. of 1989 IEEE Part. Accel. Conf., March 20-23, 1989, Chicago, IL, pp. 915-917.

[13] E.D. Courant, A.A. Garren, and U. Wienands, Low Momentum Compaction Lattice Study for the SSC Low Energy Booster, Proc. of 1991 IEEE Part. Accel. Conf., May 6-9, 1991, San Francisco, California, pp. 2829-2831.

[14] D. Trbojevic, D. Finley, R. Gerig, and S. Holmes, Design Method for High Energy Accelerator without Transition Energy, Proc. of Second European Particle Accelerator Conference, Nice, France, June 1990, pp. 1536-1538.

[15] K.Y. Ng, D. Trbojevic, and S.Y. Lee, A Transitionless Lattice for the Fermilab Main Injector, Proc. of 1991 IEEE Part. Accel. Conf., May 6-9, 1991, San Francisco, California, pp. 159-161.

[16] E.D. Courant and H.S. Snyder, Theory of the Alternating Gradient Synchrotron, Ann. Phys. 3, 1 (1958).

[17] S. Peggs, S. Tepikian, and D. Trbojevic, First Order Modification of Transition Energy at RHIC, Proc. of 1993 IEEE Part. Accel. Conf., May 17-25, 1993, Wasington, DC.

[18] S.Y. Lee, K.Y. Ng, and D. Trbojevic, A Design of a Quasi-Isochronous Storage Ring , Proc. of 1993 IEEE Part. Accel. Conf., May 17-25, 1993, Washington, DC,pp. 102-104.

[19] S.Y. Lee, K.Y. Ng, and D. Trbojevic, Design and Study of Accelerator Lattices without transition, Fermilab Internal Report FN-595, 1992.

\title{
DISCLAIMER
}

\begin{abstract}
This report was prepared as an account of work sponsored by an agency of the United States Government. Neither the United States Government nor any agency thereof, nor any of their employees, makes any warranty, express or implied, or assumes any legal liability or responsibility for the accuracy, completeness, or usefulness of any information, apparatus, product, or process disclosed, or represents that its use would not infringe privately owned rights. Reference herein to any specific commercial product, process, or service by trade name, trademark, manufacturer, or otherwise does not necessarily constitute or imply its endorsement, recommendation, or favoring by the United States Government or any agency thereof. The views and opinions of authors expressed herein do not necessarily state or reflect those of the United States Government or any agency thereof.
\end{abstract}




\section{DISCLAMMER}

Portions of this document may be illegible in electronic image products. Images are produced from the best available original document. 